\title{
The "Stick-Slip" Phenomenon During the Active Earth Pressure Failure of a Granular Soil of Analogical Material
}

\author{
Sidi Mohammed Daoud \\ Civil Engineering Dpt \\ Djillali Liabes University \\ Sidi Bel Abbes, Algeria \\ daoudmed22@yahoo.fr
}

\author{
Mourad Meghachou \\ Civil Engineering Dpt \\ Djillali Liabes University \\ Sidi Bel Abbes, Algeria \\ mourad_meghachou@univ-sba.dz
}

\author{
Abbad Hichem \\ Civil Engineering Dpt \\ Djillali Liabes University \\ Sidi Bel Abbes, Algeria \\ hi_abbad@yahoo.fr
}

\author{
Pierre Vacher \\ SYMME Laboratory \\ University of Savoie \\ France \\ pierre.vacher@univ- \\ savoie.fr
}

\begin{abstract}
The aim of this paper is to highlight the "stick-slip" phenomenon in the mechanisms of failure related to active earth pressure of an analogical material simulating a granular medium. For this, a two-dimensional small-scale model was used and a phenomenological analysis of these mechanisms was conducted employing measurements of the fields of deformations using the method of digital image correlation. It was noted that a fluctuation in the response of the material occurs during the equal increments of displacements imposed on the mobile wall. This incremental response of the medium during the failure process related to active earth pressure explains the "stick-slip" phenomenon.
\end{abstract}

Keywords- stick-slip phenomenon; image correlation; smallscale model; granular material; analogical material

\section{INTRODUCTION}

It is commonly accepted that when a shear failure is caused by active earth pressure, a line of rupture extends inside the ground and behind the plate. In the case of granular materials, this line represents the zone where mainly the slips, and consequently frictions between the grains of material, will take place. The failure of granular materials in this phenomenon is characterized by the formation of a rigid block which constitutes the moving part. This rigid block slips compared to the rest of material. One of the important occurrences of this friction is the stick-slip phenomenon.

The stick-slip characterizes a jerky movement when an object is drawn on a scraping surface via a spring. The movement of this object is generally not uniform. Schematically, periods where the object resists and does not move (stick period), and shorter periods where the resistance limit is exceeded and the object slips along an important distance (slip period), are observed [1].

The purpose of the experiment described in this paper is to cinematically study the influence of the incremental displacements of a mobile plate on the response of a granular material constituted of metallic cylindrical particles (analogical material). This way, it would be possible to highlight the stickslip movement during the active earth pressure failure.

\section{THE “STICK-SLIP” BEHAVIOUR IN GRANULAR MATERIALS}

The "stick-slip" behaviour is observed in some granular materials during the shear failure [2, 3]. During the "stick" phase, the particles at the shear interface are closely interlocked and have shear strength. The material starts then a period of dilatance, and a slip takes place between some particles.

An analysis regarding the "stick-slip" phenomenon during triaxial tests made on a glass balls material with diameters from 630 to $840 \mu \mathrm{m}$ and with various strain rates $(0.083 \mathrm{~mm} / \mathrm{min}$, $0.18 \mathrm{~mm} / \mathrm{min}$, and $0.5 \mathrm{~mm} / \mathrm{min}$ ), and related figures can be found in [4]. In addition, an acoustic measurement method allows hearing diffuse screeching during the deformation of granular materials [4]. They correspond to intermittent slips, localized atthe contacts between the grains. Another approach is to study the stick-slip phenomenon by a fluctuation in the behaviour of glass balls and sand samples [5].

\section{THE EXPERIMENTAL MODEL}

In order to reproduce the shear failure by active earth pressure of a granular soil, a small-scale model was designed, integrating a mechanism which allowed moving horizontally a vertical plate. Behind this vertical plate an analogical material made of small metallic cylinders is laid down simulating a purely granular soil.

The medium had a surface of $550 \mathrm{~mm}$ width and $300 \mathrm{~mm}$ height, dimensions allowing a semi-infinite medium. Indeed, the observed slip lines when propagating are not blocked by the boundary of the experimental model. Figures 1 and 2 illustrate the experimental device.

It has been shown that the study of mechanic problems of granular materials can be approached using a 2D device by replacing the soil by a cylindrical particles [6]. In this case, the 
cylinders are made of steel and have a $4 \mathrm{~mm}$ diameter and $70 \mathrm{~mm}$ length. The material is known as mono-disperse since only one diameter of cylindrical particles and only one material has been used. A unit weight of steel around $68.9 \mathrm{kN} / \mathrm{m} 3$ confers to the material a weighing nature. The nondeformability of the cylindrical particles simulates an incompressible material.

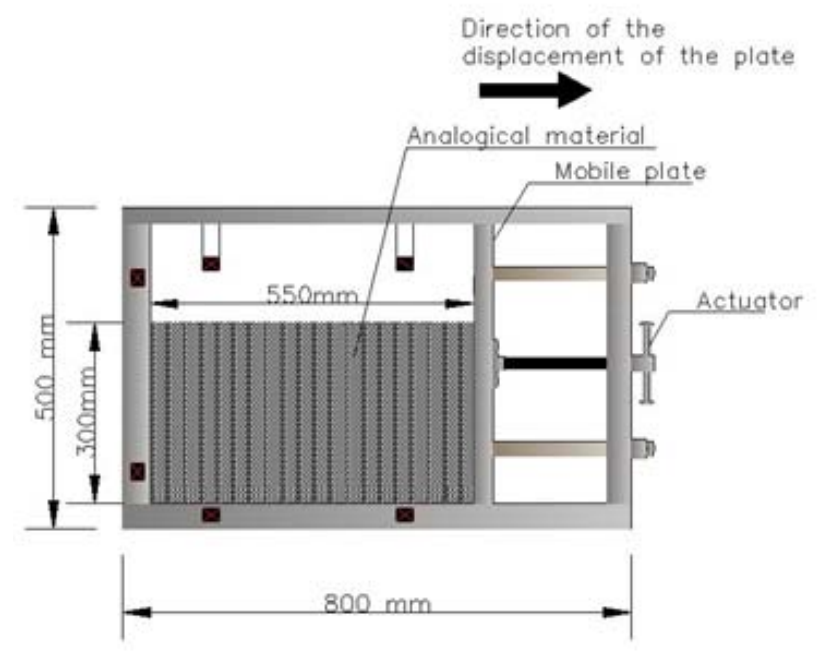

Fig. 1. Scheme of the experimental device used.

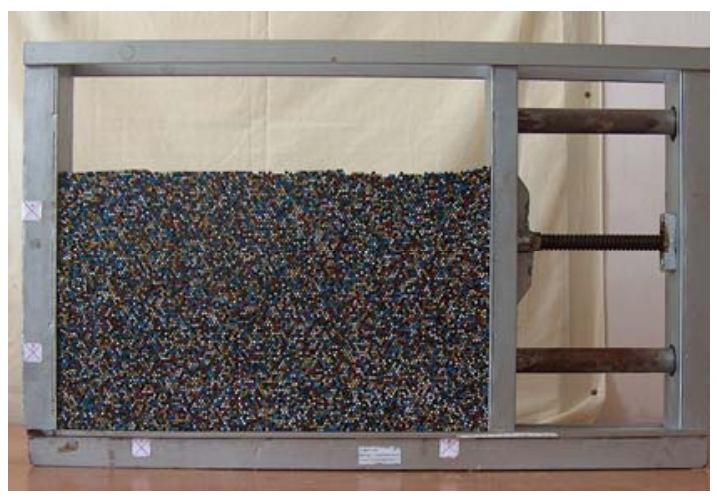

Fig. 2. Picture of the device used.

The deformations of the medium is studied from a macroscopic point of view supposing a continuous displacement field although the imposed displacement increments are discontinuous.

The laying procedure of the cylindrical particles has been set to put them always in orthorhombic contact (Figure 3). This is supposed to put the medium in a dense state. Measurements on the apparent unit weight and the absolute unit weight with respective values of $68.9 \mathrm{kN} / \mathrm{m} 3$ and $78.7 \mathrm{kN} / \mathrm{m} 3$, gives a real void ratio equal to 0.142 . In order to obtain a visual contrast, the ends of cylindrical particles were painted with 5 different colors. After each test the material was removed then replaced. That will avoid any heterogeneity of the medium and thus any localization of deformation.

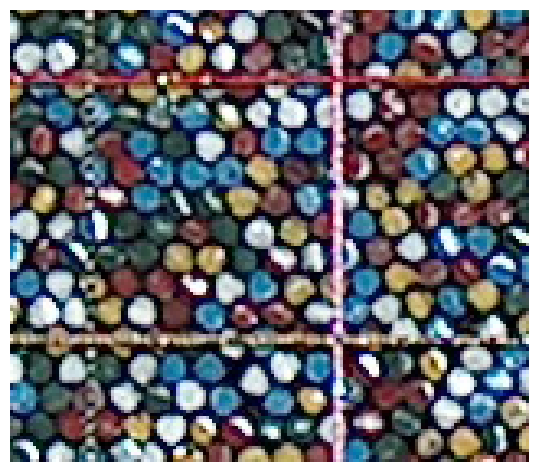

Fig. 3. Zoom on the material constituting the analogical material.

\section{DEFORMATIONS MEASUREMENT AND EXPERIMENTAL PROCEDURE}

The deformation analysis of the material will be made by a digital image correlation method using the "7D" software. 7D is a software for the analysis of digital images whose principal function is to measure displacements and to calculate deformations on plane or not plane surfaces by digital images correlation method.

Images have a resolution of $2592 * 1944$ pixels. The dimensions of the medium in pixels are $1465 * 800$ (dimension of a pixel corresponds to $0.375 \mathrm{~mm}$ ). A base of measurement of $20 * 20$ pixels was employed, which corresponds to a $7.5 \mathrm{~mm}$ point of measurement.

Uncertainties of measurement in terms of displacement are difficult to identify in the shear zones because of the discontinuities of the displacement fields (rotation of the cylindrical particles, brutal change of relative position etc). The displacement values for a zone located far from the slip surface, are approximately $0.01 \mathrm{~mm}$. The measure uncertainty in a less deformed zone is thus approximately the 1/30th of a pixel. This relatively poor uncertainty is strongly related to the quality of the camera used.

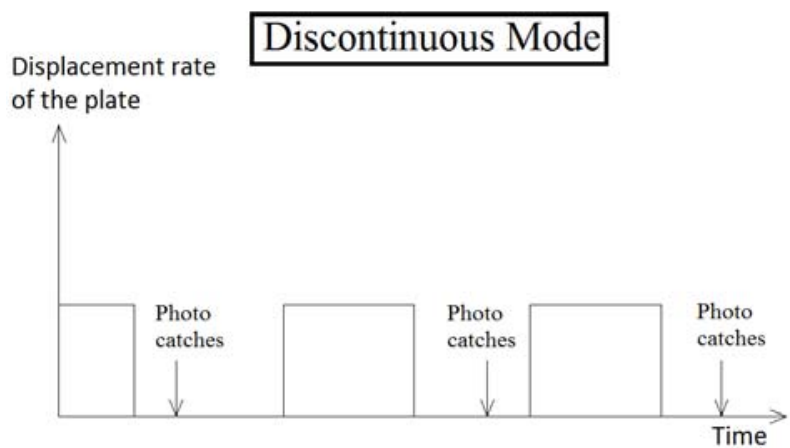

Fig. 4. Displacement rate of the mobile plate of the device and time of photo catches during the tests in discontinuous mode

The shear failure in the medium will be initiated by the vertical mobile plate (which moves horizontally). The procedure is to drive the plate at a constant speed of $0.25 \mathrm{~mm} / \mathrm{s}$ by a step of $1.5 \mathrm{~mm}$, which corresponds to a displacement of 
approximately 4 pixels on the images. The plate moves, then stops, a photo is taken, then the plate moves again etc. This mode of displacement is called discontinuous mode (Figure 4).

Once all photographs have been recorded, two options of treatment are possible. A global analysis can be done, which corresponds to comparing each deformed state with the initial state (state 1 and 2 ; state 1 and $3 ; \ldots$; state 1 and state $\mathrm{N}$ ), or an incremental analysis can be done, which corresponds to comparing each deformed state with the previous one (state1 and 2 ; state 2 and $3 ; \ldots$; state $n-1$ and state $n$ )

\section{TESTS ANALYSIS AND INTERPRETATIONS}

The displacements vectors fields for the first displacement increments of the mobile plate in incremental analysis (i.e. by comparing the results of each deformed state to the preceding one), clearly show the formation of a rigid wedge slipping compared to the rest of the medium. In order to better represent these data, a factor of amplification of $1600 \%$ was used.

The slip of this rigid wedge has a fluctuated movement although the displacement increment of the plate is identical at each stage. This is highlighted by the analysis of successive stages. In stages 2 and 3 , the maximum displacement increments are approximately 4.1 pixels, the horizontal component is well found and a weak vertical component appears (Figure 5). This stage corresponds to the stick phase where the slipping corner of material sticks to the rest of material.

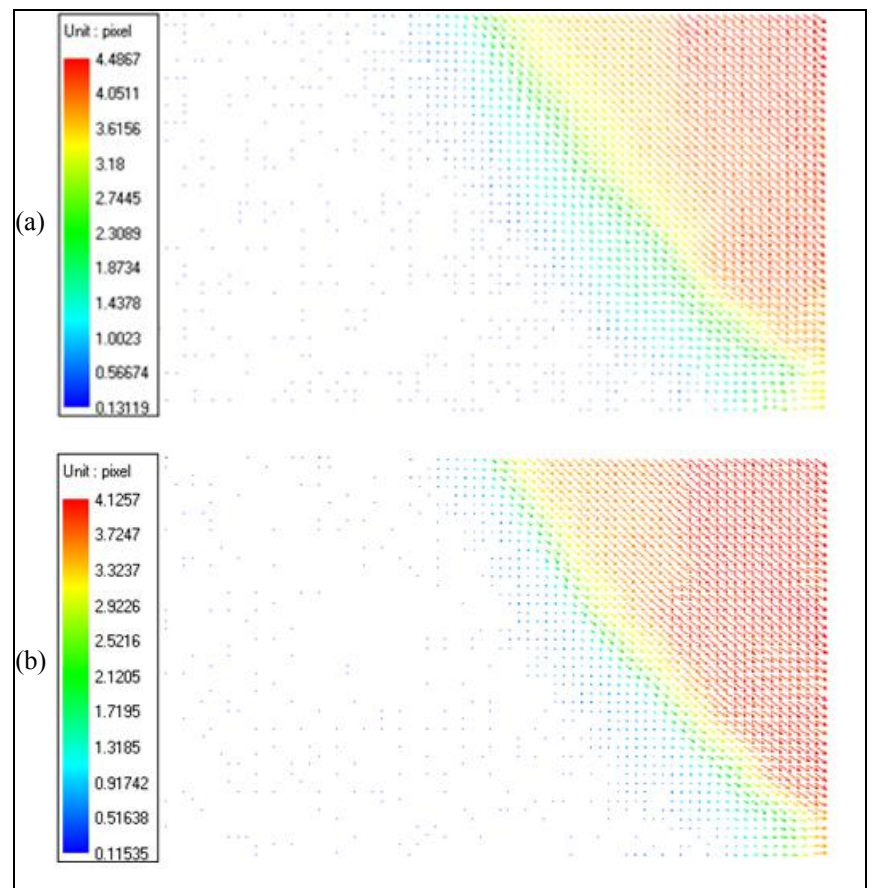

Fig. 5. Displacement increments analysis of the medium between two stages of the displacement of the mobile plate: (a) displacement of 8 pixels (3mm). (b) displacement of 12 pixels $(4.5 \mathrm{~mm})$
The movement of the wedge is more accentuated during the following phases. Beyond a displacement increment of $4.5 \mathrm{~mm}$, displacement vectors show an important and a sudden slump of the wedge with displacement increment values reaching 8.29 pixels locally, which correspond to the slip phase (Figure 6). The wedge takes again a less accentuated movement, and the displacement increments approach those of the first phase (stick phase) (Figure 7).

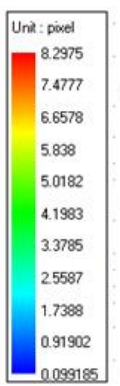

Fig. 6. Displacement increment at the fourth stage of displacement of the mobile plate corresponding to the slip phase

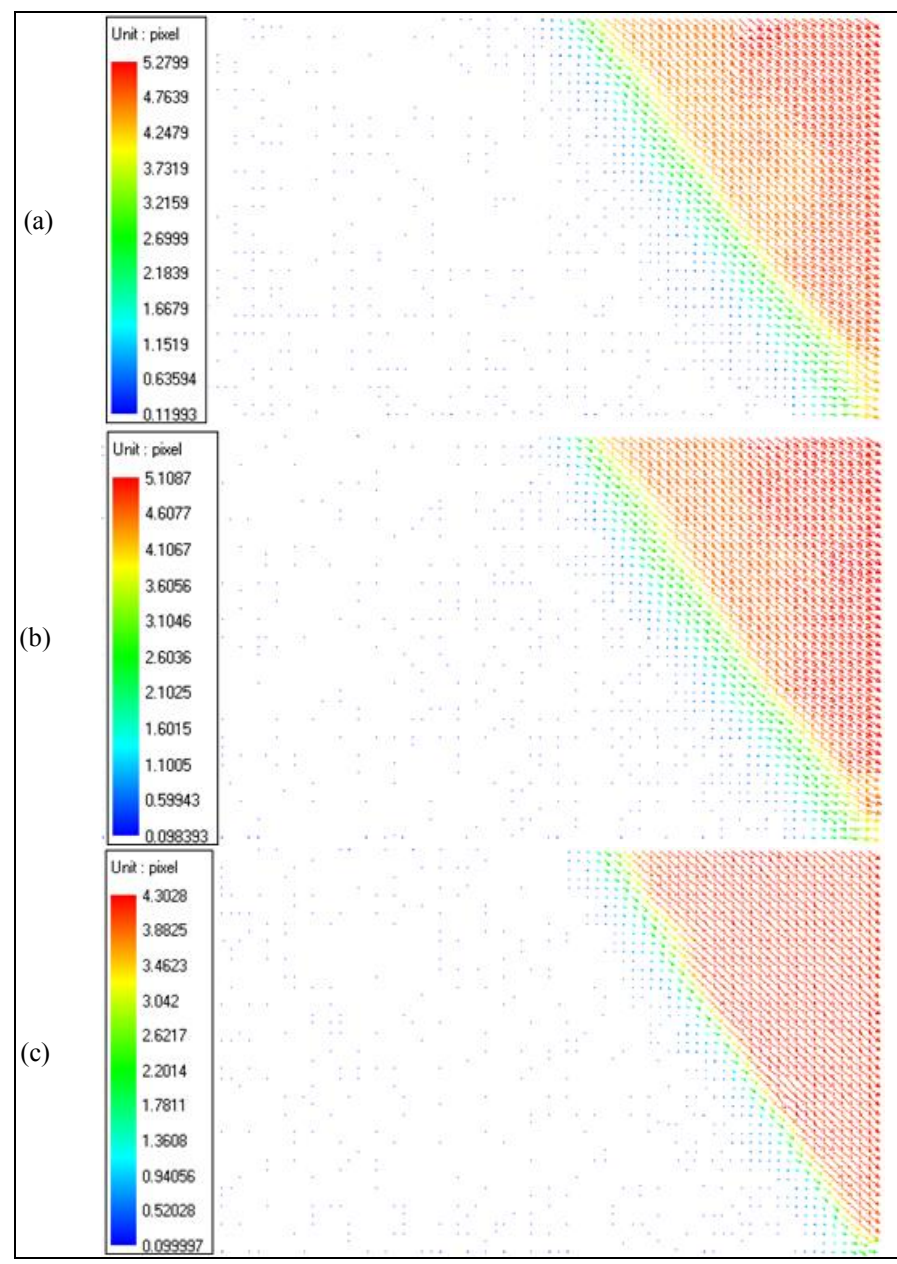

Fig. 7. Displacement increments analysis of the medium between two stages of the displacement of the mobile plate: (a) displacement of 20 pixels $(7.5 \mathrm{~mm})$. (b) displacement of 24 pixels $(9 \mathrm{~mm})$. (c) displacement of 28 pixels (10.5 mm). 
And it is only with the eighth displacement increment of the plate $(8 \times 4$ pixels $)$ that the wedge shows again an important slip, with displacement vectors reaching an important value of 17.83 pixels (Figure 8). On the graphics of Figure 9, the average of the displacements vectors measured on the entire medium for each displacement increment of the plate for three tests is depicted. Thus, fluctuations during the displacement of the rigid wedge can be noted.

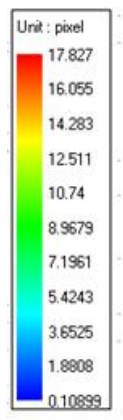

Fig. 8. Displacement increment at the eighth stage of displacement of the mobile plate corresponding to the slip phase.

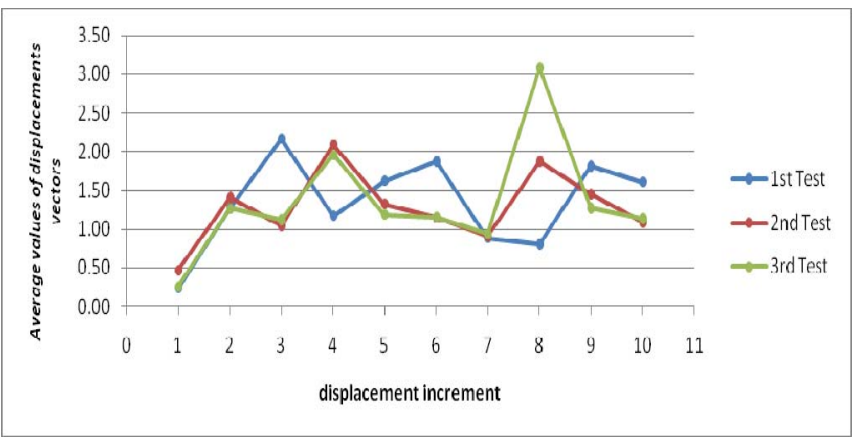

Fig. 9. Average values of displacement vectors measured for each test.

\section{CONCLUSION}

The fluctuation in the movement of the rigid wedge slipping during the failure shows the property of the granular material to adopt a stick-slip movement during the shear failure, although there are uniform displacement increments of the wall. It would be interesting for future investigations to see the influence of the rate of this increment on the material. The mode of displacement in this paper is discontinuous. It would also be interesting to see how the behavior of the material for a continuous mode of plate displacement.

\section{REFERENCES}

[1] L. Bocquet, "Approche physique du frottement", 597éme Conférence de l'Université de tous les savoirs, Paris, 2005

[2] I. Albert, P. Tegzes, R. Albert, J. G. Sample, A. Barabàsi, T. Vicsek, B. Kahng, P. Schiffer, "Stick-slip fluctuations in granular drag", Physical Review E: Statistical, Nonlinear, and Soft Matter Physics, Vol. 64, No. 3, pp. 0313071-0313079, 2001

[3] G. Ovarlez, Statique et rhéologie d'un milieu granulaire confiné, Thèse de Doctorat, Université Paris XI Orsay, 2002
[4] F. Adjemian, P. Evesque,"Experimental study of stick-slip behaviour", International Journal for Numerical and Analytical Methods in Geomechanics, Vol 28, No. 6, pp. 501-530, 2004

[5] L. E. Roussel, Experimental investigation of Stick-Slip behavior in granular materials, MSc Thesis, Civil and Environmental Engineering Department, Louisiana State University, 2005

[6] N. Dolzhenko, Etude expérimentale et numérique de modèle réduit bidimensionnel du creusement d'un tunnel. Développement d'une loi de comportement spécifique, Thèse de Doctorat, INSA de Lyon, 2005 